\title{
Las vacunas contra el Covid-19: dos dilemas éticos a considerar
}

\section{Vaccines against Covid-19: Two ethical dilemmas to consider}

\begin{abstract}
Yael Zonenszain Laiter*
https://doi.org/10.36105/mye.2021v32n1.06

\section{Resumen}

La epidemia causada por el Covid-19 ha alterado y redefinido la capacidad de los sistemas de salud a nivel mundial, al mismo tiempo que ha presentado un reto para los bioeticistas, que deben enfocarse en cuestiones como el proceso ético de desarrollo de las vacunas, y las estrategias que deben seguirse para garantizar una distribución justa y equitativa de ellas. Se analiza la estrategia de las pruebas de exposición directa al virus como un posible camino para acelerar la disponibilidad de las vacunas contra el Covid-19, y sus implicaciones éticas. También se exhibe la problemática global que se tendrá que enfrentar para asegurar una vacuna universal y accesible a todos, y algunas estrategias de distribución de las vacunas a nivel local, que aseguren un proceso ético y justo para todos los miembros de la sociedad. Se presentan diferentes posturas éticas en referencia a ambos problemas, exponiendo distintos argumentos y soluciones a los dilemas éticos planteados.
\end{abstract}

\footnotetext{
* Miembro de la Academia Mexicana de Bioética. Profesora de la Facultad de Bioética de la Universidad Anáhuac México, México. Correo electrónico: yael.zonens zainl@anahuac.mx https://orcid.org/0000-0001-6273-4921

Recepción: 20 de septiembre de 2020. Aceptación: 15 de octubre de 2020.
} 
Palabras clave: SARS-CoV-2, desarrollo de vacunas, pruebas de exposición directa, distribución ética.

\section{Introducción}

El año 2020 trajo consigo la pandemia más extensa y mortal de la que se haya tenido registro en la historia de la humanidad. El SARS$\mathrm{CoV}-2$, que se asoció posteriormente al nombre de enfermedad del coronavirus Covid-19, como manifestación clínica del mismo, vino a alterar y a redefinir la capacidad de los sistemas de salud a nivel mundial. Además, le dio un papel preponderante a la bioética, dados los dilemas derivados del manejo de la pandemia. Entre los muchos problemas que se deben afrontar, están el de la asignación de recursos escasos en el ámbito médico, el manejo ético de los pacientes y, en el caso particular de etapas posteriores, el desarrollo de medicamentos y vacunas capaces de combatir la enfermedad.

La ética en investigación aplicada al desarrollo de vacunas es un campo que ha sido analizado desde hace muchos años, y en el que están bien establecidos los protocolos que debe seguir la industria farmacéutica. La discusión se centra ahora en algunos problemas éticos que se pueden presentar durante la etapa de investigación y desarrollo de las vacunas, independientes de aquéllos ya regulados en la ética en investigación, y en los dilemas que indudablemente habrá que afrontar cuando se alcance la etapa de distribución de las mismas.

Primero, se expone de manera general cuál es el proceso que se sigue para la producción de una vacuna, desde las etapas iniciales de investigación, hasta su aprobación para ser lanzada al mercado. En la segunda parte, se analiza la estrategia de las pruebas de exposición directa al virus como un posible camino para acelerar la disponibilidad de las vacunas contra el Covid-19, y sus implicaciones éticas. Por último, se presenta la problemática global que se tendrá que enfrentar para garantizar una vacuna universal y accesible a to- 
dos, y algunas estrategias de distribución de las vacunas a nivel local, que aseguren un proceso ético y justo para todos los miembros de la sociedad.

\section{El proceso de desarrollo de las vacunas contra el Covid-19}

Las vacunas son la manera más «costo-efectiva» de controlar la pandemia de Covid-19, y los esfuerzos internacionales se han enfocado en la producción de una que pronto pueda ser distribuida a nivel mundial. En el momento de escribir este artículo existen más de 165 vacunas contra el SARS-CoV-2 que se encuentran en diferentes etapas de investigación y desarrollo. 31 de ellas ya se encuentran en las fases de pruebas en humanos (1). Los cálculos recientes indican que las primeras vacunas estarán disponibles en un periodo de entre 12 y 18 meses (2).

Para el desarrollo de este tipo de vacunas se están empleando diferentes técnicas que, en su mayoría, se enfocan en las proteínas spike, que cubren la superficie del virus, y que son las proteínas clave que permiten la entrada a las células humanas. Los distintos tipos de vacunas emplean al coronavirus completo inactivado o atenuado, al material genético viral, sea éste $A D N$ o ARN, a otros vectores virales, como el adenovirus, o a fragmentos de proteína del virus (3).

Para que el público confíe en que las vacunas contra el Covid-19 que se están desarrollando serán realmente seguras y efectivas, debe existir un proceso de vigilancia extremo, que se ajuste a los estándares éticos y científicos más elevados a nivel mundial (4). Los protocolos en este sentido son muy estrictos, y sólo aquellas vacunas, cuyos resultados puedan verificarse por organismos pares, y que transparenten sus hallazgos, deben ser las que sean aprobadas para su aplicación a la población.

El proceso de desarrollo de una vacuna pasa por varias fases. Inicia con la etapa preclínica, en la que se comprueba la respuesta 
inmune de la vacuna en modelos animales. Posteriormente se pasa a la fase 1 de seguridad, en la que se aplica la vacuna a un grupo pequeño de personas para examinar la seguridad y la dosificación de la misma, y se comprueba la estimulación del sistema inmune en los voluntarios. En la fase 2 se lleva a cabo un estudio expandido, con cientos de personas en las que se prueba la vacuna. La fase 3 corresponde a las pruebas de eficacia, en donde la vacuna se aplica a miles de personas, evaluando cuántos individuos se infectan en comparación con las personas del grupo de control que recibe un placebo. En esta etapa se comprueba si la vacuna protege contra el virus y se revela si presenta efectos secundarios que no se hayan detectado antes.

Los voluntarios a los que se les aplica la vacuna continúan con sus actividades normales, y entran en contacto con el virus de forma azarosa durante su vida cotidiana. La fase 3 es la más prolongada del estudio, y absorbe la mayor cantidad de tiempo, debido a que en ella se debe comprobar su eficacia y seguridad en un grupo grande de voluntarios, entre los cuales muchos toman sus precauciones para no contagiarse.

La mancuerna que lleva la delantera en la carrera por producir la vacuna es la de la Universidad de Oxford y la de la farmacéutica AstraZeneca, que proponen un vector viral no replicativo como modelo para su vacuna (5). Se espera que este estudio obtenga los resultados de la fase 3 a fines de 2020.

Dada la premura que presenta la pandemia de Covid-19, muchos científicos y bioeticistas han propuesto que la fase 3 del desarrollo de las vacunas se base en pruebas de exposición directa al virus, para acortar el tiempo en el que las vacunas puedan estar disponibles.

\section{Pruebas de exposición directa al SARS-CoV-2}

Las pruebas de exposición directa (buman challenge trials) son un tipo de estudio clínico, en el que los voluntarios son expuestos directa- 
mente al patógeno, para comprobar la eficacia de un medicamento o de una vacuna en desarrollo. Los protocolos convencionales del desarrollo de vacunas y medicamentos son muy prolongados, tomando por lo general varios años, especialmente en la fase 3. Esto se debe a que tiene que pasar el tiempo suficiente para que los voluntarios entren en contacto con el virus de forma natural, y se pueda determinar si la vacuna funcionó como debía, y si, entre otras cosas, produjo efectos adversos.

Con la prueba de exposición directa, el tiempo del estudio se acorta significativamente, ya que los voluntarios se exponen directamente al virus de forma deliberada, y en muy pocas semanas se puede determinar la toxicidad y respuesta inmune a la vacuna, al compararse con un grupo de control que recibe un placebo. Otras ventajas de este método son que se necesita un número menor de participantes para obtener resultados preliminares sobre su eficacia y seguridad, y que permite hacer comparaciones entre diferentes vacunas candidatas para determinar cuál es la más efectiva (4). De esta forma, se puede hacer más rápida y eficiente la respuesta a condiciones como la pandemia actual.

En el 2016, el Centro Médico de la Universidad de Leiden, en Holanda, organizó un taller internacional e independiente, para discutir las oportunidades y retos que presentan las pruebas de exposición directa. El taller estuvo conformado por investigadores clínicos, científicos, organismos reguladores y proveedores de fondos provenientes de 22 países. Durante el taller, se expuso la importancia de este tipo de pruebas para obtener información crítica acerca de los mecanismos de las enfermedades y sobre la eficacia de las nuevas vacunas. Se hizo énfasis en la seguridad de estos estudios, ya que se reduce el número de participantes y el tiempo de desarrollo de las vacunas (6).

A pesar de lo anterior, las pruebas de exposición directa presentan diversos dilemas éticos que deben abordarse antes de su implementación (7). Una de las críticas principales se centra en la posible explotación de las poblaciones económicamente vulnerables, para 
que se beneficien de participar en dichos estudios, a cambio de incentivos monetarios. Esta crítica no es exclusiva de las pruebas de exposición directa, sino que aplica también para todas las pruebas clínicas que se realizan con cualquier nuevo medicamento o vacuna.

El éxito de la investigación clínica depende de la participación de los sujetos voluntarios. De acuerdo con las guías éticas del Consejo para las Organizaciones Internacionales de Ciencias Médicas (CIOMS), los incentivos económicos o materiales son esenciales para inducir a los individuos a participar en proyectos de investigación clínica que sean inherentemente beneficiosos (8). La compensación monetaria atrae a aquellos participantes altruistas, que confían en las metas del estudio, y que poseen las características físicas que se necesitan para la investigación en particular.

Algunos autores expresan que muchos participantes buscan la forma de cubrir sus necesidades económicas a través de su participación como voluntarios en este tipo de estudios médicos. Al ofrecer incentivos monetarios como estrategia para reclutar voluntarios, se explota a los más vulnerables y pobres de la sociedad. Proponen que, para asegurar que las personas con bajos ingresos económicos participen en este tipo de estudios, a los voluntarios se les debe compensar lo suficiente para cubrir sus gastos diarios, sin ofrecerles pagos adicionales que deriven en decisiones coercitivas (9).

Adair Richards, de la Universidad de Warwick, menciona algunos otros argumentos éticos en contra de la utilización de las pruebas de exposición directa para el desarrollo de una vacuna contra el Covid-19: a) existe un riesgo significativo de muerte o de daño serio a la salud para los participantes en el estudio; este punto es debatido por otros autores; b) los experimentos pueden no resultar en una vacuna viable; c) puede ser imposible que un individuo dé su consentimiento realmente informado y libre; el participante podría estar sujeto a presión psicológica como resultado de su propio miedo, al deseo de hacer una contribución social, o a recibir presión social de sus amigos o de la sociedad para acelerar el desarro- 
llo de la vacuna; d) la conducción de estos experimentos puede dañar la reputación de la investigación y de los investigadores involucrados, lo que se reflejaría en que la confianza del público en estos estudios disminuya, y e) estas investigaciones podrían convertirse en una pendiente resbalosa, en la que se comiencen a autorizar experimentos potencialmente no éticos (10).

Aunque, en primera instancia, las pruebas de exposición directa parecieran no éticas, existe un consenso entre la comunidad filosófica de que infectar intencionalmente a los participantes en el estudio sería éticamente aceptable en ciertas circunstancias, tales como las que predominan en el diseño de los estudios actuales, y en el contexto de la gravedad de la pandemia por SARS-CoV-2 por la que se atraviesa $(6,11,12)$.

Uno de los argumentos detrás de la aprobación ética de los estudios de exposición directa para la vacuna del Covid-19 radica en que, si un voluntario es informado de forma correcta y completa acerca de los riesgos y beneficios de participar en un estudio de este tipo, sería permisible que pudiera registrarse en él. Aunque el proyecto fuera potencialmente peligroso, no obstante fuera de forma mínima, los resultados reducirían el tiempo que se requiere para desarrollar una vacuna efectiva para todos aquéllos expuestos al virus. De no acelerar el tiempo en el que una vacuna segura y eficaz esté disponible, el virus va a continuar siendo una amenaza para la población y, en especial, para los trabajadores de la salud, los adultos mayores, y aquellas personas con comorbilidades que aumenten su riesgo de no sobrevivir a la infección (11). Al hacerse un balance entre los riesgos y los beneficios de las pruebas de exposición directa, tanto para los voluntarios como para la población en general, parece ser que los beneficios superan por mucho a los riesgos.

Según el filósofo Peter Singer, debemos ser consistentes con la actitud que tenemos del concepto de riesgo. Señala que, en otras circunstancias como, por ejemplo, en la donación de un riñón, la acción se considera loable, aun cuando representa un riesgo de uno 
en 3.300 de morir como resultado de la intervención. A pesar de eso, no se prohíbe la donación de riñones. La probabilidad de que un voluntario joven y sano fallezca como resultado de una prueba de exposición directa es menor de uno en $10.000(11,13)$. Por lo tanto, los voluntarios de estos estudios deben ser reconocidos por poner en riesgo su salud para salvar a otros.

También debe considerarse que estos voluntarios han tomado la decisión de poner en riesgo su salud de forma autónoma. Como lo establece Eyal en su artículo: Las personas adultas pueden legitimar muchas intervenciones en su cuerpo y en su salud, que normalmente están probibidas, con sólo decir 'Si' con completa comprensión y voluntad (14).

Más adelante se indica cómo debe realizarse el proceso de otorgamiento del consentimiento informado, para considerarse riguroso y para que se ajuste a todas las normas éticas establecidas.

Otro argumento a favor de las pruebas de exposición directa se basa en la cantidad de vidas que pueden salvarse con una vacuna que esté disponible en poco tiempo $(12,14)$. Se calcula que, aun con las medidas de mitigación que se han implementado a nivel global, las muertes por Covid-19 alcancen varios millones en un año (15). Si se acelera el tiempo de producción de una vacuna eficaz, el número de víctimas por la pandemia podría reducirse significativamente, además de paliar los efectos sociales y económicos que han surgido como consecuencia de las medidas de distanciamiento implementadas por los distintos gobiernos.

La Organización Mundial de la Salud propone ocho criterios éticos para que los estudios de exposición directa sean aceptados, ${ }^{1}$ entre los que se encuentran los siguientes:

1. Justificación científica: debe existir una justificación científica sólida para llevar a cabo los estudios con el virus SARS-CoV-2. Esto se resume en que los resultados que se obtengan no pudieran obtenerse de manera tan eficiente o expedita con estudios basados en otros diseños que involucraran menor riesgo para los participantes (14), y que, por lo tanto, repercutan en un mayor y más pronto beneficio para la salud pública. 
2. Evaluación de los riesgos y posibles beneficios: los posibles beneficios esperados deben ser mucho mayores que los riesgos. Los riesgos y beneficios cuantificables deben ser evaluados en tres grupos principales: a) los participantes; (b) la sociedad en general, y c) aquéllos en contacto con los participantes (16).

3. Selección del lugar: los estudios deben situarse en donde la investigación pueda conformarse con los estándares científicos, clínicos y éticos más altos; deben garantizar que puedan proveer cuidado médico de alta calidad, incluyendo servicios de terapia intensiva, seguimiento de los participantes en el largo plazo, y compensación completa por cualquier daño que se presente en relación con el estudio.

4. Selección de participantes: los investigadores deben asegurarse de que los criterios de selección de los participantes limiten y minimicen los riesgos. Los estudios iniciales deberían limitarse a grupos de adultos jóvenes y sanos, de entre 18 y 30 años de edad (13), y excluir a aquellos que tengan mayor riesgo de infección por su origen, que resulten en injusticia social y explotación, o que sean vulnerables en cualquier sentido.

5. Consentimiento informado: los estudios deben incluir un proceso de consentimiento informado riguroso. El proceso debe basarse en los mejores datos disponibles acerca de los riesgos, asegurándose de que los participantes comprendan completamente toda la información relevante. El consentimiento debe reafirmarse periódicamente, cuando surja nueva información importante, para confirmar una buena comprensión por parte de los participantes y su adhesión voluntaria al estudio. Algunos autores, como Richards, proponen que los participantes tengan un periodo suficiente de tiempo para reflexionar acerca de la decisión de integrarse al estudio, después de informarse acerca de los riesgos y beneficios; además, deben tener la posibilidad de retirarse del estudio en cualquier momento, y aun así beneficiarse del mejor cuidado médico disponible (10). 


\section{Distribución ética de las vacunas}

Existe una gran expectativa, por parte de la comunidad global, en cuanto a cuándo se va a poder contar con una vacuna efectiva contra el Covid-19. Una vez que se remonten las dificultades clínicas, técnicas y éticas de la investigación para su desarrollo, vendrá una segunda etapa en la que se presentarán conflictos con la distribución y la administración, y el acceso justo a toda la población.

Las diferencias inherentes entre los países en vías de desarrollo y aquellos desarrollados, presentan una desigualdad de base para todo el proceso de accesibilidad justa de la vacuna a todo el mundo. Hay factores nacionalistas, geográficos y comerciales que dificultarán el acceso equitativo a todas las poblaciones, tanto en tiempo como en volumen (17). Los gobiernos probablemente den prioridad a sus propias poblaciones, dadas las altas inversiones que han hecho en los procesos de producción, tanto de las vacunas, como de los medicamentos contra del Covid-19.

El 24 de abril de 2020, la Organización Mundial de la Salud (OMS), en conjunto con algunas organizaciones humanitarias y del sector privado, reafirmó su compromiso de garantizar el acceso justo y global a vacunas seguras, de buena calidad, efectivas y accesibles, contra el Covid-19 y, por tanto, de asegurar que en la batalla contra el Covid-19, nadie se quede atrás (18).

El desarrollo y distribución masiva de una vacuna efectiva es de interés global. Sin el acceso a ella, el SARS-CoV-2 seguirá circulando libremente por el planeta, empeorando las graves consecuencias de salud, sociales y económicas que ya han permeado de forma importante a nivel mundial. Los gobiernos son quienes mayores incentivos tienen para colaborar en planes multinacionales, cuando la investigación se lleva a cabo en un país y la manufactura de las vacunas se produzca en otro. De esta manera, la cooperación global es necesaria e imperativa. La distribución se debe guiar por información precisa acerca del tamaño y el perfil de riesgo de las poblaciones afectadas; por la capacidad de cada país de implementar 
campañas de inmunización, y por los datos de monitoreo epidemiológico, ya que el éxito depende de que los países recolecten y compartan los datos relevantes (19).

Como ejemplo de lo anterior, está la reciente noticia de la invitación de Rusia a México para que forme parte de la fase 3 de la vacuna Sputnik contra el Covid-19. La participación de México será a través de la inoculación de entre 500 y mil voluntarios. Esta colaboración forma parte de la estrategia de colaboración internacional, mediante la cual se garantizará que México tenga acceso oportuno a vacunas que prueben ser efectivas y seguras (20).

Algunos autores proponen cuatro principios, bajo los cuales se lleva a cabo la distribución de vacunas en la actualidad: a) habilidad de desarrollar y de comprar: aquellos países que producen las vacunas, o que tienen el poder adquisitivo para comprarlas, son los que las reciben; b) reciprocidad: en muchos casos, los países en desarrollo participan en el proceso de producción de las vacunas, pero no se benefician de ellas; c) habilidad para la implementación de programas masivos de vacunación: dado que el proceso de vacunación masiva de la población involucra ciertas consideraciones técnicas, como el transporte especializado, suficiente personal entrenado y una infraestructura de salud sólida, las vacunas se distribuyen en aquellos países en los que se pueden maximizar los beneficios y se reduce el desperdicio de este recurso escaso, y d) justicia distributiva para los países en desarrollo: aunque este principio requiere la distribución equitativa de los recursos escasos, las condiciones de la pandemia actual hacen que esta distribución no sea óptima o, incluso, que sea imposible, por los principios antes mencionados (21).

$\mathrm{Si}$ se examinan los principios expuestos arriba, se esperaría un proceso poco ético y dispar en la accesibilidad de los países pobres y emergentes a una posible vacuna contra el SARS-CoV-2. Por lo tanto, debe existir un marco de gobernanza que promueva el acceso equitativo a una vacuna para el Covid-19 y que cuente con la confianza de la comunidad internacional. En este marco, debe evitarse 
cualquier tipo de influencias políticas y comerciales. Para la instauración de este marco, es necesaria la coordinación de diversas instituciones, inversionistas, gobiernos y empresas farmacéuticas. Asimismo, la OMS debe tener un papel central para implementar los acuerdos, con base en su experiencia y credibilidad en la promoción del acceso equitativo a las tecnologías médicas. También deben involucrarse las farmacéuticas y aquellas entidades que apoyan la distribución de este tipo de tecnología en los países en vías de desarrollo, como Gavi, ${ }^{2} \mathrm{CEPI}^{3}$ y el Fondo Mundial (19). Tanto Gavi como el Fondo Mundial tienen una política análoga de elegibilidad de los países, con base en la clasificación del ingreso y el peso de la enfermedad. Como ejemplo, el 7 de agosto de 2020 se hizo pública la colaboración entre SII -la manufacturera de vacunas más grande del mundo-, Gavi y la Fundación Bill \& Melinda Gates, para acelerar la producción y distribución de hasta 100 millones de dosis de vacunas contra el Covid-19 en países de ingresos bajos y medios (24).

Es importante garantizar un buen mecanismo de financiamiento para la producción y distribución de las vacunas en los países en vías de desarrollo. Uno de los mecanismos que se pueden emplear es el de los compromisos de compra anticipada (CCA), supervisados por la OMS. Este mecanismo asegura un mercado viable para la vacuna en cuestión, una vez que esté desarrollada. Los fondos podrían provenir tanto de los gobiernos como de contribuciones filantrópicas, como es el caso de México, con el convenio entre la Fundación Carlos Slim y la farmacéutica AstraZeneca. En este caso, el acuerdo garantiza la producción y distribución, sin beneficio económico en América Latina, de la vacuna desarrollada por la farmacéutica, con una disponibilidad inicial de 150 millones de dosis (25).

Según Bollyky, Gostin y Hamburg, los beneficios de obtener una vacuna conllevan ciertas obligaciones. Dentro de estas obligaciones, se mencionan: a) el compromiso de participar en la colaboración científica; b) transparencia; c) compartir los datos y las muestras biológicas; d) compartir los datos acerca de la seguridad y la eficacia de la vacuna; e) los gobiernos deben comprometerse a eliminar 
las restricciones de exportación de las vacunas, y f) el compromiso de garantizar una distribución equitativa de la vacuna. De esta forma, se asegura un producto accesible para los grupos marginales y más vulnerables, distribuido con base en las necesidades de salud pública de cada país, y no en su capacidad adquisitiva.

Otro tema que compete a la bioética es la distribución justa de las vacunas dentro de una población, una vez que ya estén disponibles en el mercado. ¿Qué criterios deben emplearse para suministrarlas? ¿Qué personas deben recibirla primero dentro de una población? ¿Quiénes tienen prioridad, y por qué?

Las vacunas tienen la particularidad, a diferencia de los medicamentos, de que no sólo protegen a quienes las reciben, sino que también protegen a quienes rodean a esa persona. La mayoría de los bioeticistas coinciden en que el personal de salud que se encuentra en la primera línea de batalla contra el Covid-19, y otros trabajadores esenciales, como camilleros, personal de limpieza en los hospitales, etcétera, deben ser los primeros en tener acceso a la vacuna. Los trabajadores sanitarios y otros trabajadores que proveen servicios fundamentales constantemente están expuestos al virus, y al tener el derecho a trabajar en las condiciones más seguras posibles, también tienen el derecho a tener un acceso prioritario a la vacuna. Además, pueden ser un foco de infección para otros; al vacunarlos, se vela también por la salud de los pacientes con los que entran en contacto (26).

Los criterios que se utilizan con mayor frecuencia para la distribución de recursos escasos en el ámbito de la salud son la maximización de la utilidad esperada, y el principio de justicia distributiva, dando prioridad a aquéllos con necesidades mayores $(27,28)$. Bajo esos principios, los adultos mayores y las poblaciones vulnerables con otras morbilidades asociadas serían los segundos beneficiados con la vacuna.

Por lo general, la postura anterior en cuanto al orden de distribución de la vacuna es la que mayor consenso alcanza. Sin embar- 
go, algunos expresan la postura opuesta, explicando que quienes deben recibir la vacuna son los niños, para maximizar los beneficios de la inmunidad indirecta para los adultos mayores y para otros grupos vulnerables y con comorbilidades; se trata de vacunar a los jóvenes para proteger a los viejos y a los enfermos (29). Se basan en que el fin de la emergencia se va a alcanzar cuando exista una política adecuada de vacunación que alcance a las personas de la manera más efectiva, y no cuando se tenga una vacuna. La efectividad del programa se mide con el número de muertes que se puedan prevenir. Esta propuesta depende del tipo de vacuna que se desarrolle, y de los efectos adversos y efectividad que demuestre, tanto en los niños como en los adultos mayores.

\section{Conclusión}

La aparición de enfermedades nuevas nos lleva a considerar acercamientos no convencionales para solucionar los problemas que se van presentando. La pandemia causada por el Covid-19 no ha sido la excepción.

Independientemente de la ciencia, que se ha visto retada a encontrar los medicamentos y las vacunas que enfrenten la enfermedad generada por el SARS-CoV-2, la bioética ha tomado un papel preponderante durante la pandemia. Ya no es sólo cuestión del tipo de molécula que se desarrolla, sino de cuáles son los caminos correctos para hacerlo, con el fin de hacer el mejor bien para todos. Esto aplica tanto para los procesos de desarrollo de las posibles vacunas, como para la distribución justa y equitativa de dicho recurso a nivel global. La esperanza se centra, entonces, en encontrar la mejor, desarrollada de la mejor manera, y con la mayor accesibilidad para todos. 


\title{
Notas bibliográficas
}

\begin{abstract}
${ }^{1}$ Para revisar los ocho criterios éticos para los estudios de exposición directa propuestos por la Organización Mundial de la Salud, así como los potenciales riesgos y beneficios para la sociedad, para los participantes y para terceros en contacto con ellos, ver (4).

${ }^{2}$ Gavi, the Vaccine Alliance, es un proyecto mancomunado entre la Organización Mundial de la Salud, UNICEF, el Banco Mundial y la Fundación Bill \& Melinda Gates. Su misión es la de salvar vidas, reducir la pobreza y proteger al mundo de las amenazas de epidemias. También ha ayudado a vacunar a más de 760 millones de niños de los países más pobres del mundo, previniendo más de 13 millones de muertes (22).

${ }^{3}$ CEPI (Coalition for Epidemic Preparedness Innovations) es una alianza global que fue lanzada en 2017, con el fin de desarrollar vacunas que combatan futuras epidemias. Junto con Gavi y la OMS, lanzaron COVAX, para asegurar el acceso equitativo de vacunas contra el Covid-19 y, así, terminar con la fase aguda de la pandemia para finales de 2021. En la actualidad, cuenta con el portafolio más grande de vacunas potenciales, y con la participación de 172 economías a nivel mundial, para proveer el acceso equitativo y seguro de las vacunas, una vez que estén aprobadas (23).
\end{abstract}

\section{Referencias bibliográficas}

1. Corum Jonathan, Grady Denise, Wee Sui-Ly, Zimmer Carl. Coronavirus Vaccine Tracker. 2020. [Consultado el 18 de agosto de 2020]. Recuperado en: https:// www.nytimes.com/interactive/2020/science/coronavirus-vaccine-tracker.html 2. Spinney L. When will a coronavirus vaccine be ready? The Guardian. 2020; 0406. T08:55:36.000Z.

3. Pandey SC, Pande V, Sati D, Upreti S, Samant M. Vaccination strategies to combat novel coronavirus SARS-CoV-2. Life sciences (1973). $1^{\circ}$ de septiembre de 2020; 256: 117956. https://doi.org/10.1016/j.Ifs.2020.117956

4. Georgina Casey. A vaccine for Covid-19? Nursing New Zealand (Wellington, N.Z: 1995). $1^{\circ}$ de junio de 2020; 26(5): 29.

5. ISRCTN. ISRCTN89951424: A phase III study to investigate a vaccine against Covid-19. [Consultado el 18 de agosto de 2020]. Recuperado en: http://www.isrctn. com/ISRCTN89951424

6. Roestenberg M, Mo A, Kremsner PG, Yazdanbakhsh M. Controlled human infections: A report from the controlled human infection models workshop, Leiden University Medical Centre 4-6, May 2016. Vaccine. 2017; 35(51): 7070-7076. https:// doi.org/10.1016/j.vaccine.2017.10.092 
7. Mateus B. Human challenge trials are being pushed to develop a vaccine against the coronavirus. [Consultado el 20 de agosto de 2020]. Recuperado en: https://www.wsws.org/en/articles/2020/05/05/vacc-m05.html

8. Sketcher B. Guidelines. West Auckland, New Zealand. Beth Sketcher; 2018.

9. Grethlein J, Huitink L, Tagliabue A. Introduction. 2020.

10. Richards AD. Ethical guidelines for deliberately infecting volunteers with Covid-19. Journal of medical ethics. Agosto de 2020; 46(8): 502-504. https://doi.org/ 10.11 36/medethics-2020-106322

11. Singer P. The Case for Human Covid-19. Challenge Trials by Peter Singer \& Isaac Martínez. 2020. [Consultado del 18 de agosto de 2020]. Recuperado en: https://www.project-syndicate.org/commentary/ethics-of-human-covid19-challenge-trials-by-peter-singer-and-isaac-martinez-1-2020-08

12. Meagher KM, Cummins NW, Bharucha AE, Badley AD, Chlan LL, Wright RS. Covid-19 Ethics and Research. Mayo Clinic Proceedings. Junio de 2020; 95(6): 1119-1123. https://doi.org/10.1016/j.mayocp.2020.04.019

13. Verity R, Okell LC, Dorigatti I. Estimates of the severity of coronavirus disease 2019: a model-based analysis (2020). $1^{\circ}$ de abril de 2020; vol 20; 669.

14. Eyal N, Lipsitch M, Smith PG. Human challenge studies to accelerate coronavirus vaccine licensure. Marzo de 2020.

15. Walker P, Whittaker C, Watson O, Baguelin M, Ainslie K, Bhatia S, et al. Report 12: The global impact of Covid-19 and strategies for mitigation and suppression. 2020.

16. Why scientists hope to inject some people with Zika virus. [Consultado del 19 de agosto de 2020]. Recuperado en: https://www.npr.org/sections/health-shots/ 2016/02/25/468120804/why-scientists-hope-to-inject-some-people-with-zika-virus 17. Mullard A. Covid-19 vaccine development pipeline gears up. The Lancet (British edition). 6 de junio de 2020; 395(10239): 1751-1752. https://doi.org/10.1016/s01406736(20) 31252-6

18. Commitment and call to action: Global collaboration to accelerate new Covid19 health technologies. [Consultado el 25 de agosto de 2020]. Recuperado en: https://www.who.int/news-room/detail/24-04-2020-commitment-and-call-to-actionglobal-collaboration-to-accelerate-new-Covid-19-health-technologies

19. Bollyky TJ, Gostin LO, Hamburg MA. The equitable distribution of Covid-19 therapeutics and vaccines. JAMA: The Journal of the American Medical Association. 7 de mayo de 2020; 323(24): 2462. https://doi.org/10.1001/jama.2020.6641

20. Vacuna contra Covid-19. Ya hay invitación de Rusia a México para participar en vacuna «Sputnik V». 2020. [Consultado el 7 de septiembre de 2020]. Recuperado en: https://www.eluniversal.com.mx/na cion/vacuna-contra-Covid-19-ya-hayinvitacion-de-rusia-mexico-para-participar-en-vacuna-sputnik-v. https://doi.org/10. 24215/166965 81e471

21. Liu Y, Salwi S, Drolet BC. Multivalue ethical framework for fair global allocation of a Covid-19 vaccine. Journal of Medical Ethics. Agosto de 2020; 46(8): 499-501. https://doi.org/10.1136/medethics-2020-106516 
22. Gavi, the Vaccine Alliance. [Consultado el 12 de agosto de 2020]. Recuperado en: https://www.gavi.org/

23. 172 countries \& multiple candidate vaccines engaged in Covid-19 vaccine global access facility. [Consultado el 25 de agosto de 2020]. Recuperado en: https:// cepi.net/news_cepi/172-countries-multiple-candidate-vaccines-engaged-in-Covid19-vaccine-global-access-facility/. https://doi.org/10.1021/cen-09819-buscon4

24. Up to 100 million Covid-19 vaccine doses to be made available for low, and middle-income countries as early as 2021. [Consultado el 25 de agosto de 2020]. Recuperado en: https://www.gavi.org/news/media-room/100-million-Covid-19-vaccine-doses-available-low-and-middle-income-countries-2021. https://doi.org/10. $1136 / \mathrm{bmj} . \mathrm{m} 3857$

25. AFP, Redacción, Ciudad de México y Buenos Aires, Uno TV y Fundación Carlos Slim acuerdan con AstraZeneca producción de vacuna contra Covid-19 para América Latina. 2020. [Consultado el 7 de septiembre de 2020]. Recuperado en: https://www.unotv.com/nacional/astrazeneca-y-fundacion-carlos-slim-produciranvacuna-contra-covid-19-para-america-latina/ https://doi.org/10.18235/0002587

26. Malm H, May T, Francis LP, Omer SB, Salmon DA, Hood R. Ethics, pandemics, and the duty to treat. The American Journal of Bioethics. 23 de septiembre de 2008; 8(8): 4-19. https://doi.org/10.1080/15265160802317974

27. Parfit D. Another defence of the priority view. Utilitas. Septiembre de 2012; 24(3): 399-440. https://doi.org/10.1017/s095382081200009x

28. Emanuel EJ, Persad G, Upshur R, Thome B, Parker M, Glickman A, et al. Fair allocation of scarce medical resources in the time of Covid-19. N Engl J Med. 2020; 382(21): 2049-2055. https://doi.org/10.1056/nejmsb2005114

29. Giubilini A, Savulescu J, Wilkinson D. Covid-19 Vaccine: Vaccinate the young to protect the old? Journal of Law and the Biosciences. 26 de junio de 2020. https://doi. org/10.1093/jlb/lsaa050 
